\title{
Folena e Migliorini
}

\author{
Massimo Fanfani
}

PUBBLICATO: 31 MAGGIO 2021

S

e non ci fosse stata la guerra, quasi sicuramente Folena non si sarebbe laureato con Migliorini, bensi a Pisa, come tutti i normalisti. Alla Normale Folena era rimasto affascinato soprattutto da Pasquali, e Pasquali era stato la calamita che lo aveva attratto a Firenze. Nel capoluogo toscano, del resto, si recava sovente presso una zia alla quale, lui orfano di madre, era assai affezionato, come lo era della nonna materna, nella cui villa Alberti di San Giuliano allora viveva. E, fino ai suoi ultimi anni, a Firenze Folena restò sempre legatissimo: non in modo vago, ma ai tanti volti concreti che la città seppe mostrargli nel corso del tempo. Non solo quello intravisto nella sua giovinezza insieme a Pasquali, ma anche quelli del dopoguerra, legati al suo rapporto con Migliorini o all'amicizia con Giuseppe De Robertis che, nel periodo in cui fu suo assistente "a mezzo servizio", spesso riaccompagnava da San Marco alla casa di via San Gallo: "Una città sobria, minima e circoscritta come da una siepe invisibile, sede di un passeggiare riposato, ma con accensioni e scoppi improvvisi come il suo parlare, cosi diversa per esempio dalla Firenze che conoscevo attraverso Pasquali, a me forse più familiare. Per me ci sono state tante Firenze quante i maestri che ho conosciuto".

Avanti la guerra, lui ancora normalista, era stato tuttavia Pasquali, non solo a trascinarlo a Firenze, ma a suggerirgli di seguire le lezioni di Migliorini nel corso del I939-1940, che, se la data è esatta, fu il primo della nuova disciplina, la Storia della lingua italiana, che lo studioso rodigino era stato chiamato a insegnare in Italia: «conservo gli appunti sulla lingua di Galileo, lessi il primo fascicolo di Lingua nostra e il suo primo libro, ed ebbi da lui una schedina che ho conservato anchessa con un pacco di mie, un primo tema di esercitazione: "La negazione intensiva in italiano: il tipo non me ne importa un fico". Era la vigilia della guerra, di li a poco ci si sarebbe tutti dispersi, e a qualcuno potrà sembrare un po' buffo che uno studente a quei di esercitasse il suo ingegno su particolari marginali di lingua».

A testimonianza di quel primo impatto fiorentino di Folena c'è anche una foto del I94I, che fu pubblicata in un settimanale del 1943 a corredo di un articolo in cui Eugenio Montale celebrava, con una punta d'ironia, il "filologo soprano" Giorgio Pasquali fresco di nomine accademiche, descrivendo fra l'altro la sua biblioteca, una "caotica e pur ordinatissima città di libri che tanti dei suoi prediletti studenti di Firenze e normalisti di Pisa conoscono". 


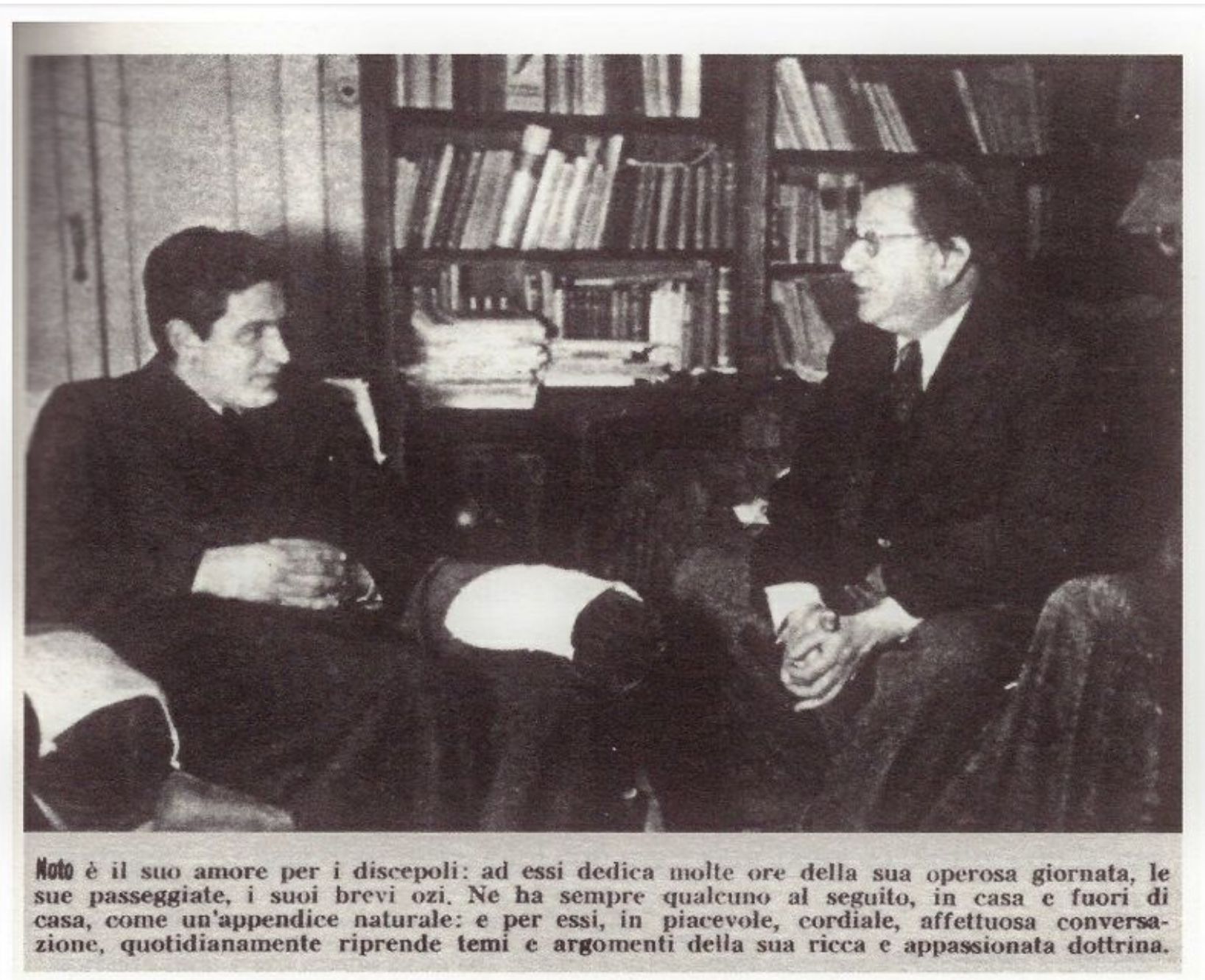

Dopo la guerra, reduce dalla prigionia, Folena avrebbe conosciuto anche l'ospitale biblioteca di Migliorini: "Credo che per tutti noi l'immagine più viva e familiare di Migliorini si inquadri indissolubilmente nella cornice della sua biblioteca [...]: la biblioteca comoda e stipata dello studio e dell'anticamera di via Lamarmora I7, con le finestre aperte sul tranquillo ordine botanico del Giardino dei Semplici. Quanto abbiamo imparato con lui là dentro. E mi avveniva di confrontarla spesso con l'altra biblioteca privata che frequentavo, quella di Pasquali, erta lassù perigliosamente sul lungarno Vespucci, a picco sull'Arno e il Ponte alla Carraia. Due biblioteche, le più vive e aperte che mi sia dato di ricordare, che più diverse nell'aspetto esterno e nell'interna composizione non si potrebbero immaginare, e dove si praticavano con ritmo opposto due modi di ricerca non comparabili, ma in una cosi profonda e feconda intesa e cooperazione".

Se Pasquali era stato una calamita, va detto che Firenze già di per sé in quegli anni esercitava una notevole forza d'attrazione per chi si occupava di linguistica e di filologia. Specie dopo che vi erano approdati Devoto nel 1935 e Migliorini nel i939. In particolare quest'ultimo, senza proclami e clamore, ma in modo semplice e pacato, aveva portato una tale ventata di interessanti novità che oggi, col senno del poi, appaiono ovvie, ma che allora, come ebbe a dire Folena, "suonavano eretiche per molti glottologi, e potevano anche costare una cattedra". Come in effetti era successo: Migliorini, fra i linguisti della sua generazione, era stato l'ultimo, e con un qualche ritardo, a salire in cattedra. Tuttavia, "poco incline a mettersi in mostra e cosi restio ad accettare responsabilità che non nascessero dalla sua vocazione", ora che gli era offerta quella possibilità, era li ad avviare il primo corso di Storia della lingua italiana, "dando subito, per noi, alla nuova disciplina storica, un volto cosi 
limpido e onesto, che pareva voluto e dettato ipsis rebus, dal suo stesso soggetto, ma era tutto suo e forse irripetibile".

L'arrivo a Firenze di Migliorini, un uomo schivo e profondamente buono, capace di suscitare interessi impegnandosi a fondo per concretizzarli e di valorizzare le risorse di ciascuno coinvolgendolo nel lavoro comune, volle dire anche l'apertura di diversi nuovi e operosi cantieri: la rivista "Lingua nostra", il progetto di un dizionario etimologico, la collaborazione con l'Enciclopedia italiana e con l'Eiar, il rifacimento del vocabolario del Cappuccini, i neologismi per le appendici al Panzini, lo scavo per la futura Storia della lingua. Cantieri in cui collaboravano studenti e studiosi delle più varie discipline.

Fra questi anche Devoto e Pasquali i quali, fino a quel momento, non si erano mai occupati di questioni relative all'italiano (e Pasquali, a dire il vero, nemmeno di linguistica). E invece cominciano entrambi, proprio adesso sul finire degli anni trenta, a trattare di lingua italiana e di questioni contemporaneistiche, specialmente sulle colonne di "Lingua nostra", spesso stimolati dagli interventi miglioriniani. Si capisce quindi perché Pasquali spingesse Folena a frequentare un uomo cosi fattivo e generoso di sé. E fu appunto su "Lingua nostra", nel r94I, che apparve anche il primo articolo del giovane studioso.

Ma, come s'è accennato, se non ci fosse stata la guerra, Folena si sarebbe laureato con altri maestri. Invece, quando nel I946, dopo la prigionia, tornò a Firenze, "una città spettrale", dei suoi vecchi maestri non trovò quasi più nessuno. Pasquali era ricoverato per una grave forma di esaurimento nervoso. Barbi, con cui aveva cominciato a lavorare prima di partire soldato, era morto. Devoto era preso in un vortice di incarichi pubblici e d'impegni politici. La fascistissima Facoltà di Lettere d'una città che era stata fra le più fasciste d'Italia, fra epurazioni regolamenti di conti casi incresciosi, era solo macerie.

In quella Firenze "spettrale" c'era comunque una persona "limpida e onesta" cui potersi rivolgere per concludere rapidamente gli studi interrotti, come fecero diversi altri studenti sbandati dalle vicende della guerra. "Fu Migliorini - scrive Folena - con la sua pacifica comunicativa fiducia nel lavoro collettivo, a ricondurmi fuori dal tunnel in cui mi trovavo. Senza di lui, probabilmente, non avrei rimontato la china".

Così Folena si laureò in Storia della lingua italiana in quello stesso I946, con una tesi sull'Arcadia del Sannazaro, che nel 1952 fu il suo primo libro. E divenne subito l'assistente di Migliorini, fin quando, alla metà degli anni cinquanta, fu chiamato alla cattedra di Padova. Per Folena, gli anni fiorentini furono decisivi nel suo percorso di maturazione umana e scientifica. In particolare, la collaborazione con Migliorini fu assai più profonda e fruttuosa di quel che poté risultare all'esterno. Migliorini stava allora ultimando il lavoro preparatorio per l'annunciata Storia della lingua e nel metterne a punto i vari capitoli molto si avvalse di quel suo straordinario scolaro. Già la tesi del 1946 sul Sannazaro rientrava in tale prospettiva di mutua collaborazione. Sul Quattrocento volgare, in passato sempre condannato da puristi e classicisti e poi trascurato dagli studiosi, si sapeva ben poco, mentre Migliorini intendeva valorizzarlo sul piano linguistico come il momento in cui si affermano in Italia le prime koinè e comincia l'espansione di una lingua comune. Proprio perciò quel periodo storico andava indagato a tappeto, negli autori toscani e nei non toscani, nei testi letterari e in quelli di carattere pratico. Ecco cosi, in quegli anni, oltre alla tesi di Folena, tutta una serie di tesi analoghe assegnate da Migliorini ai suoi scolari: su Boiardo, Alberti, san Bernardino, Ghiberti, Poliziano e su vari altri argomenti quattrocenteschi. Ecco cosi l'originale iniziativa che intraprese con Folena di pubblicare le due raccolte di Testi non toscani del Trecento (I952) e di Testi non toscani del Quattrocento (I953). 
A Migliorini, l'idea di realizzare una storia della lingua era maturata già negli anni venti - come osservò proprio Folena - e l'aveva elaborata e raffinata lungamente con una serie di saggi in cui, esaminando e approfondendo questioni e momenti particolari, riaffioravano di continuo riflessioni di teoria e di metodo non prive di rilievo, anche se espresse in modo sommesso. Infatti va ricordato, con le parole di Fiorelli, che Migliorini "aveva una istintiva ritrosia a impelagarsi in questioni teoriche".

Nel clima positivo della ripresa postbellica, mentre lavora fianco a fianco con Folena, Migliorini decide tuttavia di uscire allo scoperto con due importanti iniziative. Riunisce insieme i saggi più significativi sui criteri relativi alla storiografia linguistica - quelli in cui pur trattando aspetti particolari il suo metodo appariva più netto - nel volume Lingua e cultura del I948: basta scorrerne le pagine per ritrovare i diversi aspetti teorici della sua disciplina lucidamente esaminati sul banco di prova di singole questioni concrete. Dalla prolusione programmatica al corso di cui fu incaricato all'Università di Roma nel I931, Storia della lingua e storia della cultura, sull'importanza dei fattori culturali nell'evoluzione linguistica; a quella tenuta nel 1939 all'Università di Firenze, Correnti dotte e correnti popolari nella lingua italiana, sulla necessità di considerare i rapporti diastratici. Dalla delimitazione dell'oggetto da indagare: Lingua letteraria e lingua dell'uso del I942; a una prima trattazione dei processi che portarono dai volgari antichi a una lingua letteraria comune, studiati in un'area particolarmente interessante: Dialetto e lingua nazionale a Roma del 1932.

E insieme, in quello stesso I948, pubblica una prima sintetica ma ben articolata Storia della lingua italiana nel secondo volume dei Problemi ed orientamenti di lingua e di letteratura italiana, curati da Attilio Momigliano per l'editore Marzorati. Anche se la materia non è disposta per secoli, ma secondo una suddivisione per categorie complessive (storia esterna, storia interna, grammatiche, vocabolari, a cui seguirà nel I949, nel terzo volume dei Problemi ed orientamenti, il capitolo sulla "questione della lingua"), si tratta nella sostanza di un'anticipazione cospicua - in tutto sono I7o fitte pagine - delle idee e delle strutture portanti che si ritroveranno poi nel grande volume del ig60.

Insomma Migliorini può già allora mostrare criteri, piano, e una prima trattazione in scala ridotta dell'impresa a cui sta mirando. Nello stesso tempo, anche grazie all'aiuto di Folena, comincia a stendere e perfezionare i vari capitoli dell'opera maggiore, facendone subito materia dei suoi corsi - ci restano le dispense del 1952-1953, Tra il latino e l'italiano. Primordi della lingua italiana (476-960), che più o meno corrispondono ai capitoli II e III della Storia della lingua - e poi anticipandone una buona parte in rivista durante quel decennio.

In quegli anni di cosi fervida operosità e di grandi speranze - gli anni della ricostruzione, della modernizzazione della società, del rinnovamento della vita culturale - sembrava che non vi fossero più grossi ostacoli per poter finalmente "dare all'Italia un'opera che fino allora mancava". I due studiosi di cui ci occupiamo, l'uno prossimo a coronare il sogno della sua vita e l'altro intento a toccare una serie di significativi traguardi che lo resero presto noto come uno dei più fini filologi del momento, avevano affrontato quell'impegno senza scoraggiarsi "di fronte all'immane vastità del lavoro". In realtà, dietro alle apparenze e alla loro ammirevole abnegazione, tutto era assai più complicato e quelli che a posteriori sembrano facili successi, richiesero una notevole dose di fermezza e di sopportazione.

Ciò che forse c'inganna di più nel comprendere come stessero realmente le cose è l'atteggiamento pacato e positivo di Migliorini, un uomo del tutto alieno dalle polemiche, e dunque poco incline a mettere in piazza le tensioni che si erano scatenate intorno alla sua disciplina. Che invece fu subito contrastata e proprio nei suoi fondamenti teorici, con ripercussioni che gravarono non solo sulla collocazione e le sorti di una materia nuova e ancora priva di una sua riconosciuta identità, a cavallo com'era fra dialettologia, linguistica e stilistica - e dunque, sul piano accademico, in bilico fra la 
Letteratura italiana e la Glottologia - ma anche sull'idea stessa che allora e in seguito si ebbe dell'Italia linguistica e della sua storia.

Per capire la situazione, basta solo considerare la discussione che si accese intorno a uno degli aspetti teorici che riguardano una "storia" della lingua. Fra le varie questioni che Migliorini aveva cercato fin dall'inizio di mettere in chiaro, c'era quella, fondamentale, dell'oggetto stesso da trattare, ovvero della materia linguistica da considerare in una storia della lingua. Dato che le lingue e i dialetti presenti nel territorio italiano sono stati e sono numerosi e lo stesso "italiano", nella sua concreta realtà è vario, stratificato, cangiante, di che lingua si sarebbe dovuta ricostruire la storia scrivendo una "storia della lingua italiana”?

Nel caso specifico dell'italiano, infatti, la cosa implicava decisioni delicate e impegnative più che per altre lingue, tanto che Migliorini più di una volta tornò a riflettere su questo problema. Comunque già nella prolusione del I93I aveva sostenuto che occorreva orientarsi verso quel "quid medium" che è la lingua comune: lo storico della lingua ha per compito lo studio della "formazione della lingua comune". E di conseguenza occorreva lasciar da parte la lingua letteraria, le tante varietà parlate, i particolari idioletti, dato che l'indagine storico-linguistica non poteva ridursi all'analisi stilistica propria dei letterati o al comparativismo dei linguisti e dei dialettologi. Certo la "lingua media" che è all'intersezione delle tante varietà dell'italiano è solo un'astrazione. Ma questa astrazione è indispensabile allo storico se vuol essere obiettivo e mirare al nucleo fondamentale, quello che gli consentirà poi di valutare meglio le eccentricità e gli scarti: ovvero le varietà della lingua, le altre lingue, le scelte individuali.

Queste cose Migliorini le aveva ripetute più volte e si potevano leggere in quasi tutti i suoi libri come, ad esempio, in apertura di Lingua contemporanea (1938): "considereremo principalmente la lingua come media [...], occupandoci delle peculiarità individuali solo in quanto trovino un'eco nella lingua normale: non c'importa, insomma, in questa sede, la lingua di d'Annunzio, ci interessano i dannunzianismi della lingua di oggi". Questa chiara idea, organicamente unitaria e compatta, della lingua oggetto d'indagine storica poneva lo storico della lingua su un piano diverso da quello su cui operavano coloro che s'interessavano ai dialetti o ai fatti di stile e alla lingua dei letterati. Fra questi lo stesso Devoto il quale, non appena Migliorini lo ebbe coinvolto nei suoi progetti, si esercitò in analisi stilisticogrammaticali di letterati "eccentrici" come Gadda, e nel I940 contrappose alla concezione miglioriniana della storia linguistica una sua Storia della lingua di Roma che, come osservò bene Folena, era "concepita come storia di istituti linguistici", dove "le personalità maggiori sono presenti in quanto rappresentative della collettività", risultando comunque preponderanti.

In quella Storia di Devoto è importante soprattutto l'appendice teorica nella quale invece della lingua media, o del conguaglio delle singole parlate locali verso una lingua comune, si propone un modello più complesso e dunque più intrigante. Un modello articolato in una pluralità di aspetti linguistici distinti e contrapposti, fra i quali predomina quello letterario. La lingua per Devoto è "qualcosa di multiforme che deve essere analizzato e scomposto nei suoi elementi costitutivi". E tali elementi sono riferiti "crocianamente" a quattro poli fondamentali, "focolai che emanano tipi linguistici caratteristici" in opposizione fra loro: la "lingua letteraria" rispetto a quella "usuale", la "lingua tecnica" rispetto a quella "espressiva". E tale pluralità non si riscontra solo all'interno della lingua, ma anche in quei contesti in cui siano compresenti lingue, varietà, dialetti diversi.

Tale visione frammentata e dialetticamente articolata della storia, che invece di cercare la sintesi o il denominatore comune di una realtà tendenzialmente convergente, privilegia il mutevole gioco delle sue varie tonalità, e addirittura delle varie componenti del suo eventuale tessuto plurilingue, era 
indubbiamente affascinante e più stimolante della paziente ricerca storicistica sui nodi sottostanti all'ordito comune. Tuttavia essa venne sviluppata da Devoto solo dopo che nel I948 Migliorini ebbe pubblicato Lingua e cultura e il suo panorama di storia dell'italiano. Nel I95I, infatti, lo studioso ligure ripropose in modo più approfondito la sua originale teoria in un volumetto che costituisce il momento più alto della sua riflessione, Fondamenti della storia linguistica, dedicandolo a un altro grande studioso, Terracini, che in quegli stessi anni stava percorrendo una strada storico-stilistica parallela. Pur dialogando con quest'ultimo, Devoto aveva tuttavia come bersaglio, mai direttamente indicato, il lineare storicismo linguistico miglioriniano. La storia della lingua, per Devoto, sarebbe legittimata solo dalla presenza di una "stilistica delle scelte", che era il suo particolare modo d'intendere la stilistica di Bally.

Il nome di Migliorini è quasi del tutto ignorato anche nel Profilo di storia linguistica italiana che Devoto pubblicò nel i953. Soltanto nelle integrazioni inserite nell'Appendice all'edizione del i964, dove si parla del "fondamentale" libro di De Mauro apparso l'anno avanti (la Storia linguistica dell'Italia unita che fin dal titolo ben si accordava con quel Profilo), della Storia della lingua italiana apparsa nel I960 qualcosa si dice: "Per ragioni di principio, chiaramente spiegate, il Migliorini non si occupa di tradizioni stilistiche, e, su questo terreno, il presente Profilo rimane solo". In effetti quel libro veleggiava da solo a gonfie vele, sia quando apparve, che dopo il ig6o. Tanto che lo stesso Migliorini, per un momento, fu in dubbio se non farsi da parte: "Quando nel 1953 è uscito il Profilo di storia linguistica italiana di Giacomo Devoto, mio sodale in tante altre imprese, mi sono domandato se quello scritto, così intelligente e così suggestivo, rendesse inutile il mio".

In effetti, fin dal suo primo apparire, lo straordinario quadro storiografico devotiano, dove "l'orizzonte collettivo della dialettologia e quello delle lingue individuali della stilistica si saldano in una lucida sintesi", suscitò ammirazione ed ebbe una forte eco favorevole specie fra coloro che intendevano la storia linguistica sotto il segno della stilistica, come Fubini, Segre, Contini. E soprattutto nell'ambiente fiorentino, come testimoniano le entusiastiche recensioni di Caretti, Cecchi, Pieraccioni, Nencioni, Chiappelli.

Fra queste recensioni, quella che scavò più a fondo nel valutare la portata e la novità del metodo devotiano, fu quella di Folena: tanto che, più che una recensione, è un vero e proprio saggio: Il metodo di Giacomo Devoto dalla stilistica alla storia linguistica. Si tratta di uno scritto importante non solo per l'intelligente analisi del libro preso in esame, ma per comprendere la posizione e le idee dello stesso Folena come storico della lingua, costituendo, in sostanza, un atto di fede devotiana da parte di uno studioso di scuola miglioriniana. Ovvero una sorta di conversione sulla via di Damasco a una concezione più modernamente inclusiva e progressista della storia linguistica: "Devoto ha da tempo varcato i limiti della sociologia saussuriana, e se non concepisce certo l'istituto come somma meccanica degli individui che lo rappresentano, non lo concepisce neppure come realtà trascendente rispetto agli individui, ma sintesi di tradizioni operanti in una pluralità di formazioni storiche (e quindi lotta di classi sociali): che è lontanissimo dalla visione saussuriana di una langue sincronicamente indifferenziata, l'enorme serbatoio memoriale, potenziale che la parole attualizza. La nozione di scelta, alla quale è evidentemente ormai subordinata quella di "innovazione", introduce felicemente nella linguistica devotiana il concetto di libertà che divide il campo linguistico istituzionale in due settori, uno di opposizioni nette e l'altro di scelte virtuali, disponibili per l'individuo linguistico: il confine fra questi due settori, mobile nel tempo e nella varietà delle reazioni individuali, descrive la storia della lingua. La storia linguistica è per Devoto storia di necessità e libertà insieme".

Una tale analisi fu apprezzata da Devoto, che ebbe buon gioco di servirsene per ribadire il 
superamento dello storicismo miglioriniano giudicato come pura ricostruzione evenemenziale: "Alle pagine dedicate al Profilo Gianfranco Folena ha voluto mettere il titolo Il metodo di Giacomo Devoto. Gliene sono grato. Contrariamente a quello che si crede (e contrariamente all'opinione dell'amico e collega [Wartburg] a cui il Profilo è dedicato), il metodo nelle scienze storiche non corrisponde né a grucce né ad altre armature esterne, che, giunti a maturità o a guarigione, lasciamo da parte, o smontiamo. Il metodo è concreto e non astratto, interno e non esterno al ricercatore; è consapevolezza, e quindi risultato di un'analisi sul già fatto, non strumento per il fare. La differenza fra una storia linguistica così intesa e la filologia sta in questo: che la indagine filologica è tutta concentrata sul fatto [...]; mentre l'interpretazione storico-linguistica consiste in un dialogo tra i fatti linguistici nel loro insieme accertati, e l'Autore che vi reagisce in modo sempre vario, ora più sottomesso, ora più altero. Anziché di "metodo" si dovrebbe parlare, a proposito di questo Profilo, di "metodi": che sono tanti quante le affermazioni di cui si compone".

Un tale "altero" personalismo, come quello auspicato e rappresentato da Devoto, e soprattutto la sua proposta di pluralismo metodologico ebbero notevole presa e, anzi, subito prevalsero su altri possibili modelli utilizzabili nella ricostruzione diacronica. Tanto che innescarono quel vasto processo di rinnovamento della storiografia dell'italiano come storia delle "varietà", che produrrà frutti notevoli negli anni successivi, fino alle grandi realizzazioni storico-linguistiche dei nostri giorni. Ma condussero anche a una serie di insidiose aporie, e soprattutto a nuovi luoghi comuni e a stagnazione d'idee, in una crisi latente della disciplina che perdura tuttora.

Una crisi di cui uno studioso attento e responsabile come Folena si accorse ben presto, come traspare dallo scritto con cui nel 1976 commemorò Devoto e più ancora nell'intervento del I977, La storia della lingua oggi, una sorta di pubblica confessione di chi, ripercorrendo le tappe della sua vita e del suo lavoro di storico, si trova a fare i conti con il disgregarsi di quel terreno conoscitivo, ma anzitutto umano e civile, su cui lui stesso si era formato e che aveva contribuito non poco a dissodare: "uno storico della lingua oggi si trova in crisi. Io dovrei parlare anche della mia crisi, ma parlerò soprattutto di quella della disciplina che insegno". E dopo essersi soffermato sul glorioso decennio 1953-1963 che aveva visto l'apparizione del Profilo, della Storia della lingua e della Storia linguistica dell'Italia unita, soggiungeva: "quel decennio che coronava un lungo processo di studi, almeno ad un addetto ai lavori come me, appare in un certo modo remoto se non del tutto passato, revoluto. Infatti ci sono stati vari fatti concomitanti che, se non hanno arrestato il corso degli studi di storia della lingua, hanno notevolmente spostato l'interesse fondamentale verso altre zone della linguistica".

La crisi, per la verità, riguardava non solo la storia della lingua, ma l'intera vita culturale italiana e ha avuto effetti che continuano a gravare sul presente. Se non si può tornare al mondo di ieri e nemmeno accontentarsi della luce che ancora emana da opere come quelle ricordate da Folena, la vicenda che si è cercato di tratteggiare qualcosa riesce comunque a trasmettere per guardare con fiducia al futuro. Qualcosa che non riguarda la scienza i libri le teorie, ma gli uomini: proprio la difficile strada verso chi ci ha preceduto, per comprenderne in modo veritiero e spregiudicato la vicenda, è infatti la stessa che consente di procedere avanti senza troppi sbandamenti.

Migliorini, nonostante Folena avesse ben presto compiuto delle scelte diverse dalle sue, mai ostacolò il suo antico scolaro, ma anzi continuò a sostenerlo e a incoraggiarlo, seguendo con partecipazione il suo cammino e considerandolo uno dei più fini e profondi storici della lingua: un vero maestro da cui anche lui, come soleva dire, aveva sempre da imparare. Proprio per questo l'aveva voluto accanto a sé in tante imprese, fino ai suoi ultimi anni, quando il loro rapporto divenne ancor più intenso e familiare. 
Da parte sua, Folena nutrì per il maestro un affetto sconfinato, che crebbe proprio quando Migliorini come storico della lingua venne progressivamente emarginato; un affetto che prorompeva in modo sincero e toccante ogni volta che s'incontravano: "lui - ricordava Folena - era cosi intero, così semplice e uno nella sua rara e grande dottrina, e cosi sobrio in tutto, cosi selettivo e unitario nei suoi interessi e nella sua attività scientifica [...]; era il più capace di spersonalizzarsi, nello stesso modo come nella storia della lingua egli considerava l'individuo solo in quanto si fa società, diventa media e tradizione comune". Proprio perciò, tali uomini seppero sospingere altri uomini ad andare più avanti di loro: proprio perciò la loro storia nutre anche il nostro presente.

\section{Cita come:}

Massimo Fanfani, Folena e Migliorini, "Italiano digitale", XVII, 2021/2 (aprile-giugno)

DOI: 10.35948/2532-9006/2021.7529

\section{Copyright 2021 Accademia della Crusca}

Pubblicato con licenza creative commons CC BY-NC-ND 